\title{
Prognostic factors for recovery in Portuguese patients with Bell's palsy
}

\author{
Margarida Ferreira ${ }^{1,2}$, João Firmino-Machado33, Elisa A. Marques, ${ }^{4,5}$, Paula C. \\ Santos $^{1,6}$, Ana Daniela Simões², José A. Duarte ${ }^{1}$
}

${ }^{1}$ Faculty of Sport, Research Centre in Physical Activity, Health and Leisure, University of Porto, Porto, Portugal, ${ }^{2}$ Department of Physiotherapy, CESPU, North Polytechnic Institute of Health, Gandra and Vila Nova de Famalicão, Portugal, ${ }^{3}$ Department of Public Health - Occidental Oporto, Porto, Portugal, ${ }^{4}$ Research Center in Sports Sciences, Health and Human Development (CIDESD), University Institute of Maia (ISMAI), Maia, Portugal, ${ }^{5}$ National Institute on Aging, National Institutes of Health, Bethesda, MD, USA, ${ }^{6}$ Department of Physical Therapy, School of Health Technology of Porto, Polytechnic Institute of Porto, Vila Nova de Gaia, Portugal

Objective: The main aim of this study was to identify the prognostic factors that contribute to complete recovery at 6 weeks and 6 months in patients with Bell's palsy.

Material and Methods: This is a prospective, longitudinal, and descriptive study that included 123 patients diagnosed with facial nerve palsy (FNP) at a hospital in Guimarães, Portugal. However, only 73 patients with Bell's palsy (BP) were included in the assessment of recovery at 6 weeks and 6 months. We analyzed the demographic and clinical characteristics of the patients, including sex, age, paralyzed side, occupation, previous and associated symptoms, seasonal occurrence, familial facial palsy, patient perception, intervention options, and baseline grade according to the House-Brackmann facial grading system (HB-FGS).

Results: Of the 123 cases with FNP, 79 (64.2\%) patients had BP. Age, sex, and baseline HB-FGS grades were significant predictors of complete recovery at 6 weeks. Patients with HB-FGS grade III or lower (6 weeks baseline) had significant recovery of function at 6 months.

Conclusions: Baseline severity of BP, elderly patients, and male sex were early predictors of poor prognosis. Patients with mild and moderate dysfunction according to the HB-FGS achieved significant normal facial function at 6 months. Further prospective studies with longer observation periods and larger samples are needed to verify the results.

Keywords: Facial palsy, Prognostic factors, House-Brackmann Facial Grading System measure

\section{Introduction}

The Copenhagen Facial Nerve Study defined Bell's palsy (BP) as an acute, monosymptomatic, unilateral peripheral facial paresis of unknown etiology. ${ }^{1}$ The physiopathologic mechanism responsible for BP seems to involve swelling and inflammation of the facial nerve within the bony fallopian canal, which leads to compression and ischemia of the nerve..$^{2-5}$ The etiology of BP remains unknown even today, and its diagnosis, which is still carried out by exclusion, is accurate only if no other cause of the paresis can be found. ${ }^{5}$ Recently, studies have suggested that the primary cause of BP is the reactivation of an infectious agent in the geniculate ganglia, in particular, herpes simplex virus type 1 (HSV-1). ${ }^{4}$ The most prevalent causes of secondary facial nerve palsy (FNP) are systemic and local infections, trauma, surgery, tumor, immunologic disorders, and metabolic diseases. ${ }^{2,6}$
The annual incidence of BP ranges from 20 to 30 cases per 100,000 population, with one in 60 people being affected during their lifetime. ${ }^{1,6,7}$ The peak incidence occurs between the ages of 15 and 40 years. ${ }^{1}$ Females and males are equally affected, with the highest incidence in pregnant women (45 cases per 100,000 population) ${ }^{8}$ $\mathrm{BP}$ occurs with equal frequency on the right and the left side of the face. ${ }^{1}$ The symptoms at the onset of BP include epiphora (67\%), retroauricular pain (50\%), taste disorders (34\%), and hyperacusis $(14 \%){ }^{1}$

The prognosis is good, and approximately $70 \%$ of patients with BP recover completely within 6 months without treatment. ${ }^{1,8}$ However, $30 \%$ of patients had sequelae, such as residual paresis $(29 \%)$, contracture $(17 \%)$, synkinesis $(16 \%)$, and dry eye and crocodile tears $(4 \%){ }^{1}$ The good prognosis applies to the partial dysfunction after the onset of BP and to normal functions of taste, the stapedius reflex, and lacrimal secretion. ${ }^{1,9}$ Poor prognostic factors include old age, retroauricular pain, diabetes mellitus, hypertension, non-recovery within 3 weeks, and severe 
Table 1 Differential diagnosis of Bell's palsy

\begin{tabular}{lc}
\hline Etiology & $\boldsymbol{n}(\%)$ \\
\hline Bell palsy & $79(64.2)$ \\
Trauma (skull frature, obstetric injury) & $4(3.25)$ \\
Metabolic (Diabetes Mellitus, Hyperten- & $12(16.4)$ \\
sion) & $9(12.3)$ \\
Infectious (Otitis média, Herpes zoster) & $11(15.1)$ \\
Neurologic (Stroke, Guillan-Barré Syn- & \\
drome) & $2(2.74)$ \\
Congenital & $6(8.22)$ \\
Others (Pregnancy and puerperium) & 123 \\
Total &
\end{tabular}

degeneration of the facial nerve as shown by electrophysiologic testing. ${ }^{1,8,9}$

The evaluation of treatment in the early/acute stage of BP is made difficult by the high rates of spontaneous and complete recovery. ${ }^{1}$ The literature on treatment effects is sparse and contradictory; the complete recovery of the facial nerve depends on several factors, such as the degree of injury and the correct choice of treatments. Shafshak suggested that patients with BP who do not receive appropriate treatment may suffer from incomplete recovery with physiologic and psychological complications. ${ }^{10}$ Recent guidelines recommend the use of oral corticosteroids for all patients with BP. ${ }^{11,12}$ On the other hand, evidence suggests that physical therapy has no beneficial effects in the treatment of BP. ${ }^{11}$ Nevertheless, several studies have shown benefits in the facial symmetry and quality of life of people with BP after facial exercises..$^{9,13-15}$

Despite all clinical information, the prognostic factors are usually based on electrophysiologic tests; however, these tests are not universally available and, when performed within the first weeks, are inconclusive due to Wallerian degeneration. ${ }^{16}$ There are no reliable signs or tests at the onset of palsy that can predict outcome. Consequently, we analyze clinical data to determine the prognostic factors of BP.

The main aim of the present study was to identify the prognostic factors that contribute to complete recovery at 6 weeks and 6 months in patients with Bell's palsy.

\section{Materials and methods Study population}

This prospective, longitudinal, and descriptive study was carried out among 123 patients diagnosed with FNP from January 2009 to May 2013. The study area was the city of Guimarães, a region in northern Portugal, with a population of about 159,576 inhabitants within a radius of $23.5 \mathrm{~km}$ of the hospital (Pordata, 2001). Acute health conditions were managed by the Emergency Department (ED) of Guimarães Hospital. The diagnoses were made by general practitioners or otolaryngologists, as consultants for the ED physician. At first consultation, the etiology and diagnosis of FNP were detectable, based on medical history, physical examination or specific serology, audiologic tests, and radiologic imaging techniques, when considered useful.

A total of 123 patients with FNP were treated at the department of medicine physical and rehabilitation; they were systematically referred by the ED physician of Guimarães Hospital. The second consultation took place between 3 and 10 days after the onset of BP, during which physiatrists reviewed the results of the tests requested previously, obtained medical history, and carried out physical reexamination. Of the total of 123 cases, 73 patients with first episodes of BP were prospectively analyzed in this study. All BP patients were treated with facial neuromuscular training (FNT) only or corticosteroids plus facial neuromuscular training $(\mathrm{C}+\mathrm{FNT})$ by a physiotherapist.

At baseline of this study, 44 patients were excluded because they declined to participate or had suspected central nervous system diseases, traumatic injury to the seventh nerve, recurrent FNP, antiviral therapy, metabolic and infective diseases, or a cancer condition (Table 1).

The present study was approved by the ethics committee of Guimarães Hospital. Written informed consent to participate in the study was obtained from all patients.

The clinical data and facial motor function assessments were carried out by using the patient history and the House-Brackmann facial grading system (HB-FGS). ${ }^{17}$ The HB-FGS is a widely used evaluation system and is adopted by the American Academy of Otorhinolaryngology-Head and Neck Surgery. ${ }^{17}$ The scale is used to analyze the asymmetry at rest, voluntary movement, and synkinesis. On this scale, grade I indicates normal facial function, and grade VI refers to total paralysis. In the present study, recovery was defined as the achievement of HB-FGS grade I. The patients were assessed over a period of 6 months $(t 0$, baseline; $t 1,6$ weeks; and $t 2,6$ months).

\section{Statistical analysis}

Descriptive statistics (frequencies, percentages, and means \pm standard deviations) were used for the demographic characteristics; the stratified analysis was based on the outcome of complete recovery (HB-FGS grade I) and incomplete recovery (HB-FGS grade $>$ I). The binary logistic regression was adjusted to predict complete or incomplete recovery using independent variables (age, sex, intervention type, and baseline HB-FGS grade) at 6 weeks after the onset of BP. Discrimination was measured using the area under the receiver operating characteristic (AUROC) curve, and 95\% confidence intervals (CI) were reported. The independent $t$ test and $\chi^{2}$ test were used for a comparison between complete and incomplete recovery at 6 months after the onset of BP based on the patient demographics and clinical characteristics (age, sex, HB-FGS grade at 6 weeks, and intervention type). $P$ values of less than 0.05 were considered significant. The analyses were carried out using SPSS version 22.0. 
Table 2 Demographic and clinical characteristics in patients with Bell's palsy

\begin{tabular}{|c|c|c|c|}
\hline Characteristics & Patients $(n=73)$ & Characteristics & Patients $(n=73)$ \\
\hline Sex; $n(\%)$ & & Affected side; $n$ (\%) & \\
\hline Female & $38(52.1)$ & Left & $40(54.8)$ \\
\hline Male & $35(47.9)$ & Right & $33(45.2)$ \\
\hline Age (years /mean $\pm S D$ ) & $43.64 \pm 19.2$ & $\begin{array}{l}\text { First signal of muscles contrac- } \\
\text { tion in patients with grade VI / } \\
H B-F G S \text { (day/mean } \pm S D)\end{array}$ & $18.84 \pm 15.9$ \\
\hline Occupation; n (\%) & & Patient's perception; n (\%) & \\
\hline Employed & $41(56.2)$ & Emotional stress & $15(20.5)$ \\
\hline Unemployed & $4(5.48)$ & Cold & $12(16.4)$ \\
\hline Students & $12(16.4)$ & Infection respiratory & $4(5.48)$ \\
\hline Reformed & $16(21.9)$ & $\begin{array}{l}\text { Fatigue } \\
\text { Unknown }\end{array}$ & $\begin{array}{l}1(1.37) \\
41(56.2)\end{array}$ \\
\hline Seasons of the year; $n$ (\%) & & Intervention type; n (\%) & \\
\hline Winter & $24(32.9)$ & $\mathrm{C}+\mathrm{FNT}$ & $42(57.5)$ \\
\hline Spring & $18(24.7)$ & FNT & 31 (42.5) \\
\hline \multicolumn{4}{|l|}{ Summer } \\
\hline \\
\hline Baseline of HB-FGS; $n$ (\%) & & Without symptoms; n (\%) & $9(12.33)$ \\
\hline & $8(11.0)$ & Symptoms & \\
\hline ॥ & $21(28.8)$ & Dysgeusia & $33(45.2)$ \\
\hline III & 9 (12.3) & Epiphora & $38(52.1)$ \\
\hline IV & $9(12.3)$ & Dry eye & $5(6.85)$ \\
\hline V & $26(35.6)$ & Hyperacusis & $17(23.3)$ \\
\hline $\mathrm{VI}$ & & Retroauricular pain & 25 (34.2) \\
\hline Familial facial palsy; n (\%) & & $\begin{array}{l}\text { Start of } C+F N T \text { intervention } \\
\text { (day/ mean } \pm S D \text { ) }\end{array}$ & $4.36 \pm 0.41$ \\
\hline Yes & $14(19.2)$ & $\begin{array}{l}\text { Start of FNT intervention (day/ } \\
\text { mean } \pm S D \text { ) }\end{array}$ & $5.55 \pm 0.48$ \\
\hline No & $59(80.8)$ & & \\
\hline
\end{tabular}

Note: HB-FGS, House-Brackmann Facial Grading System; FNT, facial neuromuscular training; C + FNT, corticosteroids plus facial neuromuscular training.

Table 3 Evaluations of recovery muscles in patients with VI grade of HB-FGS baseline $(n=73)$

\begin{tabular}{lcc}
\hline Facial muscles & First signal of muscles contration $\boldsymbol{n}(\%)$ & Complete recovery after $\mathbf{6}$ weeks $\boldsymbol{n}(\%)$ \\
\hline Total $(\mathrm{Vl}$ grade/HB-FGS) & $26(35.6)$ & $9(34.6)$ \\
Frontalis & $10(38.5)$ & $6(23.1)$ \\
Corrugator supercilii & $1(3.85)$ & 0.00 \\
Orbicularis oculi & $2(7.69)$ & 0.00 \\
Piramidal & $2(7.69)$ & 0.00 \\
Risorius & $9(34.6)$ & $3(11.5)$ \\
Orbicularis oris & $2(7.69)$ & 0.00 \\
\hline
\end{tabular}

\section{Results}

BP presented in 79 of 123 consecutive cases of FNP $(64.2 \%)$ in Guimarães Hospital, Portugal, during the fouryear study period (Table 1)

The mean age of the sample was 44 (range, 18-93) years, with a higher incidence observed at ages 18 49 years (unpublished data; Table 2). The occupational frequency of BP was highest among employed people, and the seasonal occurrence was during periods of extreme temperature. Regarding the affected side, 40 (54.8\%) patients presented with left facial palsy, and 14 (19.2\%) had familial palsy. The most frequent symptom was epiphora, and more than half of the BP cases had unknown causes. Applying the HB-FGS, the most frequent degree of paralysis was found to be grade VI (Table 2).

Among patients with complete paralysis, the first signal of remission occurred more frequently on the frontalis muscle and at 18 days after the onset of BP (Tables 2 and 3). Occupational status, sex, seasonal factors, affected side, first signal of remission, familial palsy, and number of symptoms (one or more) did not show an association with complete recovery outcome at all moments of evaluation $(p>0.05)$ (unpublished data).

Figure 1 shows the HB-FGS grades of the recovered patients. At 6 months of follow-up, 47 (64.4\%) patients acquired normal function (HB-FGS/grade I) at 6 weeks, and $13(17.3 \%)$ patients at 6 months. Both stratification grades had significant recovery of grade remission at 6 weeks $(p<0.001 ; t 1)$ and 6 months $(p=0.003 ; t 2)$ (Fig 1).

We identified the following factors as significant predictors of complete and incomplete recovery: age (OR, $0.91 ; p=0.012)$, baseline HB-FGS grade (OR, 0.049; $p=0.001)$, and sex $(\mathrm{OR}, 9.28 ; p=0.05)$. The predictive performance of these variables for complete/incomplete recovery was excellent, with an AUROC of 0.96 (95\% CI, 0.928-0.998). Intervention type was not a significant predictor (OR, $0.74 ; p=0.76$ ) (Table 4). 


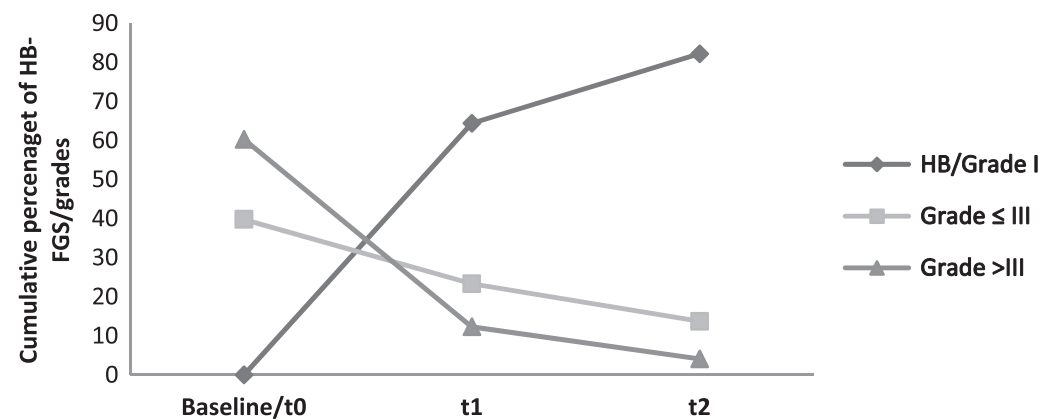

\begin{tabular}{lccc}
\hline HB-FGS $(\mathrm{n}=73)$ & Baseline (t0) & After 6 weeks (t1) & After 6 months (t2) \\
\hline HB/Grade I & - & $47(64.4)$ & $60(82.2)$ \\
Grade $\leq$ III & $29(39.8)$ & $17(23.3)$ & $10(13.7)$ \\
Grade $>$ III & $44(60.3)$ & $9(12.3)$ & $3(4.11)$ \\
\hline
\end{tabular}

Figure 1 Cumulative percentage total and stratification of complete recovery

Table 4 Predictors of complete and incomplete recovery (grade I of HB-FGS) at 6 weeks

\begin{tabular}{|c|c|c|c|c|c|}
\hline \multirow[t]{2}{*}{ Predictors } & \multicolumn{5}{|c|}{ Bell's Pals, $n=73$ patients } \\
\hline & Complete recovery $(n=47)$ & Incomplete recovery $(n=26)$ & $p$ value & Odd ratio (OR) & $95 \% \mathrm{Cl}$ \\
\hline$\overline{\text { Age } / y, \text { mean (SD) }}$ & $39.88 \pm 17.18$ & $49.16 \pm 20.21$ & 0.012 & 0.91 & $0.84-0.98$ \\
\hline \multicolumn{6}{|l|}{ Gender, No.(\%) } \\
\hline Male & $22(46.8)$ & $16(61.5)$ & 0.050 & 9.28 & $1.1-85.7$ \\
\hline Female & 25 (53.2) & 10 (38.5) & & & \\
\hline \multicolumn{6}{|c|}{ Intervention type, No.(\%) } \\
\hline C+FNT & $27(57.4)$ & $15(57.7)$ & 0.76 & 0.74 & $0.10-5.32$ \\
\hline \multicolumn{6}{|c|}{ Baseline of HB-FGS, No.(\%) } \\
\hline Grade I & $47(64.4)$ & - & & & \\
\hline Grade II & - & $7(9.6)$ & & & \\
\hline Grade III & - & $10(13.7)$ & 0.001 & 0.049 & $0.10-0.29$ \\
\hline Grade IV & - & $3(4.1)$ & & & \\
\hline Grade V & - & $4(5.5)$ & & & \\
\hline Grade VI & - & $2(2.7)$ & & & \\
\hline
\end{tabular}

Table 5 Predictors of complete and incomplete recovery between 6 weeks and 6 months

\begin{tabular}{lccc}
\hline & \multicolumn{3}{c}{ Bell's Palsy $n=26$ patients } \\
\cline { 2 - 4 } Predictors & $\mathrm{d} f$ & $\boldsymbol{X}^{2}$-value & $\boldsymbol{p}$-value \\
\hline Baseline of 6 weeks (HB-FGS/grade $\leq$ III) & 1 & 7.287 & $0.007^{*}$ \\
Interventions type & 1 & 0.004 & 0.952 \\
Sex & 1 & 0.153 & 0.696 \\
Age & 1 & 0.481 & 0.488 \\
\hline
\end{tabular}

$p<0.05$.

Patients with HB-FGS grade III or lower BP (6 weeks baseline) showed a significant association with complete recovery between 6 weeks and 6 months (Table 5).

\section{Discussion}

Occupational status, sex, season of the year, affected side, first signal of muscle contraction, familial palsy, and symptoms showed no influence on complete recovery. The predictors of good BP outcome were female sex, young patients, and slight severity at baseline according to the HB-FGS. Intervention type had no effect on recovery outcome. At 6 weeks after the onset of BP, mild and moderate dysfunction was a good prognostic factor for complete recovery within the first 6 months.

The results of our study showed that the association of genetic factor with complete recovery was not statistically significant. In contrast, Sánchez-Chapul et al. ${ }^{18}$ reported that the prognosis of complete recovery was more favorable when patients had a history of familial BP. In the present study, there was no association between side of facial palsy and complete recovery, which is in agreement with the literature. ${ }^{18,19}$ Patient's perception of cause of BP were higher in physical factor (temperature) and psychological (emotional stress). 
Laboratory and clinical studies have identified several factors that may trigger the reactivation of latent HSV-1 infections. ${ }^{20}$ These factors fall into two general categories: physical stressors (e.g. ultraviolet radiation, local trauma, coinfections, and cold) $)^{18,21,22}$ and psychological stressors (e.g. social stress and mood disorders). ${ }^{23,24}$ However, our study found no association of physical and psychological factors with complete recovery. There was also no significant association between symptoms and complete recovery. Kasse et al. ${ }^{19}$ reported that decreased tearing was related with good prognosis, whereas Adour et al. ${ }^{25}$ observed that taste disorders and decreased tearing were related with worse prognosis.

At 6 weeks of follow-up, 47 Portuguese patients showed full recovery from facial nerve paralysis; 13 other patients had similar recovery at 6 months. During this period, $10(13.7 \%)$ patients regained partial recovery from mild-to-moderate dysfunction, and 3 (4.1\%) patients achieved HB-FGS grade IV. In the literature review, the estimated percentage of complete recovery from BP was between 70 and 95\% depending on the severity and intervention type. ${ }^{1,11,26}$ In a study done in Denmark, ${ }^{1}$ all patients with BP achieved some degree of muscular function. Approximately $85 \%$ of Danish patients recovered within 3 weeks, and $15 \%$ within 3 to 5 months. ${ }^{1}$

Regarding gender, our study results showed a slightly higher incidence of BP in females, as previously reported by Kasse et al. ${ }^{19}$ We found no association between gender and complete recovery after 6 weeks, suggesting that this factor does not influence the prognosis of the disease, in accordance with previous findings in the South American population. ${ }^{19}$ Pregnancy and history of menstrual disorders seem to be a risk factor for the occurrence of BP. ${ }^{27}$ However, our study found better prognosis for females at 6 weeks, which might be explained by, among other factors, the influence of the endocrine environment, once the anti-inflammatory role have favorable effects with estrogens in many diseases. ${ }^{28}$

The effect of age on early outcome has been previously reported; so, the present study found association between aged and recovery at 6 weeks after onset of BP. Changes in recovery have been shown to be significantly diminished after 45 years. ${ }^{1,29}$ This may be due to a number of factors, including the normal loss of myelinated fibers during the aging process. $^{29}$

Our study showed that complete recovery at 6 months of follow-up was more frequent in patients with mild-to-moderate dysfunction, predicting a good prognosis. In the Asian population, severe palsy has also been reported to be a poor prognostic factor for recovery from BP. ${ }^{30}$ Another study found that the recovery rates in Asian patients with incomplete function (HB-FGS grade $\leq$ IV) were significantly higher compared with cases of severe or complete paralysis (HB-FGS/grade $\geq \mathrm{V}) .{ }^{31}$

\section{Study limitations}

The present study has a number of limitations. First, a relatively small number of cases was included in the analysis. Second, although the HB-FGS is the most widely used outcome measure of severity of palsy and has been shown to have good inter-rater reliability, its sensitivity to changes in facial symmetry is low. Third, BP was diagnosed through clinical evaluation.

\section{Conclusion}

Baseline severity of BP, elderly patients, and male sex were found to be early predictors of poor prognosis. Patients with mild and moderate dysfunction according to the HB-FGS achieved significant normal facial function at 6 months. Further prospective studies with longer observation periods and larger samples are needed to verify the results.

\section{Disclosure statement}

No potential conflict of interest was reported by the authors.

\section{References}

1 Peitersen E. Bell's palsy: the spontaneous course of 2,500 peripheral facial nerve palsies of different etiologies. Acta Otolaryngol Suppl. 2002;549:4-30.

2 Scully C, Langdon J, Evans J. Marathon of eponyms: 2 Bell palsy (idiopathic facial palsy). Oral Dis. 2009;15(4):307-308.

3 Ahmed A. When is facial paralysis Bell palsy? Current diagnosis and treatment. Cleve Clin J Med. 2005;72(5):398-405.

4 Murakami S, Mizobuchi M, Nakashiro Y, Doi T, Hato N, Yanagihara N. Bell palsy and herpes simplex virus: identification of viral DNA in endoneurial fluid and muscle. Ann Intern Med. 1996;124(1):27-30.

5 Dawidowsky K, Branica S, Batelja L, Dawidowsky B, Kovać-Bilić L, Simunić- Veselić A. Anatomical study of the facial nerve canal in comparison to the site of the lesion in Bell's palsy. Coll Antropol J. 2011;35(1):61-65.

6 Finsterer J. Management of peripheral facial nerve palsy. Eur Arch Otorhinolaryngol. 2008;265:743-752.

7 Rowlands S, Hooper R, Hughes R, Burney P. The epidemiology and treatment of Bell's palsy in the UK. Eur J Neurol J Eur Fed Neurol Soc. 2002;9(1):63-67.

8 Holland NJ, Graeme MW. Recent developments in Bell's palsy. BMJ. 2004;329(4):553-557.

9 Teixeira LJ, Soares BGDO, Vieira VP, Prado GF. Physical therapy for Bell's palsy (idiopathic facial paralysis) (Review). Cochrane Database Syst Rev. 2008;3:1-36.CD006283.

10 Shafshak ST. The treatment of facial palsy from the point of view of physical and rehabilitation medicine. Eur Medicophys. 2006;42(1):41-47.

11 Baugh RF, Basura GJ, Ishii LE, Schwartz SR, Drumheller CM, Burkholder R, et al. Clinical practice guideline: Bell's palsy. Otolaryngol Head Neck Surg. 2013;149(3):S1-S27.

12 Almeida JR, Guyatt GH, Sud S, Dorion J, Hill MD, Kolber MR, et al. Management of Bell palsy: clinical practice guideline. CMAJ. 2014;186(12):917-922.

13 Pereira LM, Obara K, Dias JM, Menacho MO, Lavado EL, Cardoso JR. Facial exercise therapy for facial palsy: systematic review and meta-analysis. Clin Rehabil. 2011;25(7):649-658.

14 Ferreira M, Santos PC, Duarte J. Idiopathic facial palsy and physical therapy: an intervention proposal following a review of practice. Phys Ther Rev. 2011;16(4):237-243.

15 McCaul JA, Cascarini L, Godden D, Coombs D, Brennan PA, Kerawala CJ. Evidence based management of Bell's palsy. Br J Oral Maxillofac Surg. 2014;52(5):387-391.

16 Hsieh R-L, Wang L-Y, Lee W-C. Correlates of degree of nerve involvement in early Bell's palsy. BMC Neurol. 2009;9(22):1-5.

17 House JW, Brackmann DE. Facial nerve grading system. Otolaryngol Head Neck Surg. 1985;93(2):146-147. 
18 Sánchez-Chapul L, Reyes-Cadena S, Andrade-Cabrera JL, CarrilloSoto IA, León- Hernández SR, Paniagua-Pérez R, et al. Bell's palsy. a prospective, longitudinal, descriptive, and observational analysis of prognosis factors for recovery in Mexican patients. Rev Invest Clin. 2011;63(4):361-369.

19 Kasse CA, Ferri RG, Vietler EYC, Leonhardt FD, Testa JRG, Cruz OLM. Clinical data and prognosis in 1521 cases of Bell's palsy. Int Congr Ser. 2003;1240:641-647.

20 Stjernquist-Desatnik A, Skoog E, Aurelius E. Detection of herpes simplex and varicella-zoster viruses in patients with Bell's palsy by the polymerase chain reaction technique. Ann Otol Rhinol Laryngol. 2006;115(4):306-311.

21 De Diego JI, Prim MP, Madero R, Gavilán J. Seasonal patterns of idiopathic facial paralysis: a 16-year study. Otolaryngol Head Neck Surg. 1999;120:269-271.

22 Spengos K, Sameli S, Stouraitis G, Kolias A, Koulouri O, Kokkinos $Z$, et al. Seasonal variation of Bell's palsy in Athens, Greece-a hospital-based retrospective evaluation over fifteen years. Eur Neurol. 2006;55(2):84-88

23 Zorrilla EP, McKay JR, Luborsky L, Schmidt K. Relation of stressors and depressive symptoms to clinical progression of viral illness. Am J Psychiatry. 1996;153:626-635.

24 Padgett DA, Sheridan JF, Dorne J, Berntson GG, Candelora J, Glaser R. Social stress and the reactivation of latent herpes simplex virus type 1. Proc Natl Acad Sci USA. 1998;95:7231-7235.

25 Adour KK, Byl F, Hilsinger RL, Kahn ZM, Sheldon MI. The true nature of Bell's palsy: analysis of 1000 consecutive patients. Laryngoscope. 1978;88:787-801.

26 Nicastri M, Mancini P, De Seta D, Bertoli G, Prosperini L, Toni D, et al Efficacy of early physical therapy in severe Bell's palsy: a randomized controlled trial. Neurorehabil Neural Repair. 2013;27(6):542-551.

27 Katz A, Sergienko R, Dior U, Wiznitzer A, Kaplan DM, Sheiner E. Bell's palsy during pregnancy: is it associated with adverse perinatal outcome? Laryngoscope. 2011;121:1395-1398.

28 Straub RH. The complex role of estrogens in inflammation. Endocrine Rev. 2007;28(5):521-574

29 May M, Shaitkin B. The facial nerve. 2nd ed. New York: Thieme; 2000.

30 Sathirapanya P, Sathirapanya C. Clinical prognostic factors for treatment outcome in Bell's palsy: a prospective study. J Med Assoc Thai. 2008;91(8):1182-1188.

31 Ikeda M, Yuzuru A, Kukimoto N, Omori H, Nakazato H, Ikeda K. Clinical factors that influence the prognosis of facial nerve paralysis and the magnitudes of influence. Laryngoscope 2005;115:855-860. 